\title{
Pharmacotherapy and Bumetanide in Autism Treatment
}

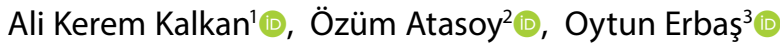

Until 50 years ago, autism spectrum disorder (ASD) was a rare childhood disease. However, today it has become a very common (one in 88 people) ${ }^{[1]}$ and a lifelong disease that is considered heterogeneous. It includes a spectrum, the severity of which can vary slightly from serious to serious, but the majority of patients need lifelong support.

Genetic studies show that autism has a strong genetic component that can involve the interaction of many different genes ${ }^{[2]}$ However, however, mutations in different genes eventually produce certain specific disorders in cognitive and social behavior. ${ }^{[3]}$

Although ASD is significantly related to heredity, the etiological causes of the disease are not understood in more than $80 \%$ of cases. ${ }^{[4]}$ Again, it remains to be discussed whether common variants in ASD occur with multiple gene interactions, major effects of rare mutations, or as a combination of both. ${ }^{[5]}$ In addition to genetic factors, many systematic reviews and meta-analysis have suggested that prenatal and postnatal factors, pregnant diet and lifestyle effects also have an effect on ASD. ${ }^{[6]}$ For example, there are studies showing that thiomersal in vaccines may have a role in the development

\footnotetext{
${ }^{1}$ Hacettepe University, Faculty of Pharmacy, Ankara, Turkey

${ }^{2}$ Department of Radiation Oncology, Dr. Lütfi Kırdar Kartal Training and Research Hospital, Istanbul, Turkey

${ }^{3}$ ERBAS Institute of Experimental Medicine, Illinois, USA \& Gebze, Turkey

Correspondence: Ali Kerem Kalkan. Hacettepe Üniversitesi Eczacılık Fakültesi, 06230 Altındağ, Ankara, Türkiye.

E-mail: alikalkan@hacettepe.edu.tr
}

Cite this article as: Kalkan AK, Atasoy Ö, Erbaş O. Pharmacotherapy and Bumetanide in Autism Treatment. JEB Med Sci 2020;1(2):52-61.

doi: $10.5606 /$ jebms.2020.75612

Received : September 06, 2020

Accepted : September 23, 2020

Published online : November 09, 2020

@2020 Journal of Experimental and Basic Medical Sciences. All rights reserved.

\begin{abstract}
Currently, autism spectrum disorder is a common, heterogeneous disease. It is diagnosed through behavioral questionnaires, parent, and doctor observations due to there are no biomarkers. The treatment is mostly carried out through education and behavioral interventions. Proven effective drugs of concomitant diseases can also be added to the treatment regimen if it is necessary. Nevertheless, recent studies have identified several compounds including arbaclofen, oxytocin, sulforaphane, and bumetanide that have an impact on the main symptoms of autism, such as social communication deficits and restrictive/repetitive behaviors. Bumetanide, a proven diuretic, is one of the most studied chemicals in the past decade. In this review; first, diagnosis of autism and tests used for it, are briefly explained, thereafter the pharmacological treatment approaches of autism, the targeted new treatment mechanisms, the association between intracellular chloride concentration with autism are mentioned respectively. After summarising the frequently used animal models, preclinical and clinical studies that focus on the effect of bumetanide, which is the center of this review, on autism were evaluated chronologically.

Keywords: Autism spectrum disorder, bumetanide, diuretic.
\end{abstract}

of autism. ${ }^{[7,8]}$ Embryonic exposure to antiepileptic valproic acid has been shown to cause in a significant percentage of patients. ${ }^{[3,9]}$ Folic acid supplementation during pregnancy has been shown to reduce the risk of ASD. ${ }^{[10]}$ These examples show that environmental factors play a role in increasing or decreasing the risk of autism.

When the neurobiology of the disease is examined; It is seen that ASD does not have a specific area of the brain. It is an event that arises mostly from the reorganization of the whole brain in the early stages of development. The findings mostly showed a general poor connection in the brain and local over-connection in some specific areas (especially the frontal and occipital lobes). ${ }^{[11,12]}$ 
Among the neurotransmitter systems, there is strong evidence that defects in the GABAergic inhibitor system are associated with autism. Autism patients are often reported to have rearrangement on chromosome 5q11-13, and this chromosome contains a set of GABA receptor genes containing GABRA5, GABRG3 and GABRB3 ${ }^{[13,14]}$ In addition to the GABA receptor genes, genes involved in the differentiation of GABAergic interneurons ${ }^{[15,16]}$ and displacement ${ }^{[17,18]}$ have also been associated with ASD. Destruction in GABAergic interneurons; Explain why epilepsy and behavioral disorders coexist in patients with ASD. ${ }^{[19]}$ Based on this information, it can be deduced that autism can be caused by disorders in the differentiation of GABAergic neurons and mechanisms in migration to the cortex. ${ }^{[3]}$

Although ASD occurs with a change in early brain development and neural reorganization, ${ }^{[12]}$ is diagnosed based on behavior, as there are no reliable biomarkers. ${ }^{[6,20]}$ In this sense, although the symptoms are varied, two important main symptoms are lack of social communication and limited, repetitive sensory-motor behavior. There may be some disorders accompanying ASD, developmental retardation, mental disability, speech difficulties and motor difficulties are examples. In addition, various forms of anxiety can be seen in ASD patients. ${ }^{[6]}$

The tests commonly used to evaluate the severity of autism and treatment response in clinical studies in autism are CARS, ABC, RRB, RDEG, ADOS G, respectively. CARS is a behavioral-rating scale and is used to evaluate the presence and severity of symptoms in ASD and to determine the effect of treatment within a certain time interval. It has a higher hit rate and less false positive identification than $A B C$ and other tests. ${ }^{[2]} A B C$ (Abnormal Behavior Checklist) is a questionnaire completed by a doctor conducting treatment during a discussion with parents. ${ }^{[2]} \mathrm{CGI}$ (Clinical Global Impressions) is used by the clinician to assess the severity of the disease, its change over time, and the effectiveness of the drug ${ }^{[23]}$ RRB (Repetitive and Restricted Behavior Scale) is a standardized checklist of 35 items on a scale of 0-5 points. Repetitive, restricted behaviors should be clearly expressed and characteristic of the person. ${ }^{[24]}$ RDEG (The Regulation Disorders Evaluation Grid) is a French activity scale that allows to determine the level of dysregulation and slowness of the babies' response. ${ }^{[25]}$ ADOS G (Autism Diagnostic Observation Program) is a standardized criterion that evaluates people suspected of having ASD in terms of social interaction, communication, creative use of games and materials. ${ }^{[26]}$

\section{Pharmacological Treatment Approaches in Autism Spectrum Disorder}

When diagnosed at an early age in autism, quite positive results can be obtained with correct interventions, because brain plasticity is open to change in early childhood, cognitive development can be achieved and the severity of symptoms can be alleviated with the applications. ${ }^{[4]}$ Therefore, it is important to be able to develop effective treatments in childhood.

Although autism is a biological disorder, basic symptoms can be improved with the help of primary education and behavioral therapies, pharmacological treatment has a more complementary role. ${ }^{[6]}$ Pharmacotherapy provides increased response to behavioral and educational interventions in this context, and in addition to a general increase in function, therefore pharmacotherapy should always be a part of disease management, which includes behavioral and educational interventions, and should focus on specific goals. ${ }^{[4]}$ Although there is such an approach in theory, FDA approved drugs for ASD are used only to treat aggression and irritability accompanying the disease, other pharmacological therapies are currently under research to improve the symptoms of ASD.

There are many drugs that are claimed to treat ASD-related behavior. These; Atypical antipsychotics, propranolol, oxytocin, vasopressin antagonists, arbaclofen, bumetanide and sulforafan. ${ }^{[27]}$ For example, in randomized controlled trials, risperidone and aripiprazole, among atypical antipsychotics, improved irritability and agitation in children and adolescents. However, both drugs caused adverse events such as sedation and weight gain ${ }^{[6]}$ Arbaklofen is a GABA-B agonist. In BTBR mice, it increased repetitive behaviors such as spontaneous grooming and cluster burial while increasing social interaction. A number of ASD-related social disorders have also improved, and clinical trials in patients with fragile $X$ syndrome also appear to have a good effect on stereotypical behavior and a tendency to limited attention. ${ }^{[28,29]}$ Following sulforafan therapy, other ASD-related behaviors such as anxiety, depression, learning, and memory were evaluated in mice.

Long-term treatment reduced anxiety-related behaviors and depression-related behaviors in mice with sciatic nerve damage. ${ }^{[30]}$ Again in an 18 -week study with humans, an improvement in social interaction, 
abnormal behavior and verbal communication was observed after sulforafan treatment. ${ }^{[31]}$ Antiepileptic drugs and selective serotonin reuptake inhibitors have again shown promising results, but there are no specific indications yet. ${ }^{[4]}$ Metformin helps improve weight gain due to antipsychotics in ASD. ${ }^{[32]}$ About autistic children.

In $30-80 \%$, attention deficit and hyperactivity disorder (ADHD) accompanies ASD and this affects both behavioral interventions and the quality of life of patients. Among the ADHD drugs, methylphenidate and guanfazin may be effective against problems caused by hyperactivity and short attention span. ${ }^{[3,34]}$ On the other hand, sleep problems from comorbid conditions have been reported in $43-83 \%$ of children with ASD, which is associated with increased behavioral and psychological problems. ${ }^{[35]}$ It has been reported that administration of melatonin is safe in such a situation; When applied, it improves sleep parameters, reduces stress and is reflected in daily behavior. ${ }^{[36]}$ Clonidine has also been shown to be effective in sleep disorders. ${ }^{[37]}$

\section{Targeted New Treatment Mechanisms}

The main symptoms of autism are due to disorders in the brain circuits and synaptic neuronal interactions that develop in early childhood. ${ }^{[4]}$ Many studies have shown the relationship between autism and serotonergic, ${ }^{[38]}$ dopaminergic ${ }^{[39]}$ GABAergic ${ }^{[40]}$ and glutamatergi $\mathrm{i}^{[4]]}$ pathways. Some of the clinical symptoms are indicative of dysfunction in these neurochemical signal systems. Therefore, selective pharmacological interventions on neurobiological pathways may play an effective role in the treatment of autism. For example, risperidone is effective on the dopaminergic system. It creates significant positive effects in children and adolescents who experience behavioral problems such as irritability, aggression, self-harm and anger attacks. ${ }^{[42]}$ Drugs acting on glutamate receptors can alter the course of the disease by acting where NMDA receptor stimulations are excessive. In a double-blind study, antiviral and antiparkinsonian NMDA receptor antagonist amantadine showed a significant effect in improving the initial hyperactivity and inappopriate speech. ${ }^{[43]}$ Abnormally low plasma oxytocin values have been reported in children with autism in relation to high oxytocin precursor levels. This suggests that autism may be related to the abnormal synthesis of the hormone in question. ${ }^{[44]}$ In a 2010 study, when intranasal oxytocin was administered in a small sample with autism, social behavior was observed to improve, thereby suggesting that oxytocin may affect the main symptoms of autism. ${ }^{[45]}$

In the light of the information revealed by the results of genetic studies, voltage-gated calcium channels are among the candidate targets that are effective in autism spectrum disorder. Both genetic and functional data show that voltage-gated calcium channels play a potential role in autism spectrum disorder. ${ }^{[5]}$ High levels of $\mathrm{Ca} 2+$ increase aspartate/ glutamate carrier activity, accelerate mitochondrial metabolism, thereby increasing oxidative stress in autistic brains. Aspartate/glutamate transporter and altered $\mathrm{Ca} 2$ + homeostasis play a role in increasing signaling events leading to autism. If these mechanisms are targeted, new therapeutic strategies can be produced. Treatments that manipulate extracellular $\mathrm{Ca} 2+$ entry or intracellular $\mathrm{Ca} 2$ + release from the endoplasmic reticulum are in principle available. ${ }^{[46]}$ Of these, $\mathrm{Ca}+2$ chelation is not recommended as a form of treatment since it causes a serious side effect such as hypocalcemia. ${ }^{[47,48]}$ The drug named ziconotide used in another approach is a synthetic peptide that selectively blocks $\mathrm{N}$-type voltage-sensitive calcium channels. For the release of neurotransmitters, calcium flow through these channels is required at the presynaptic terminals of neurons. Inhibition of $\mathrm{N}$-type calcium channels by ziconotide blocks the calcium entering the neuron. Thus, the release of neurotransmitters, such as glutamate, into the synaptic cavity is reduced. This mechanism may set new goals to alleviate autism symptoms. ${ }^{[49]}$

GABAergic signals also change in autistic patients. Thus, a change in the excitation/inhibition rate is observed. ${ }^{[50]}$ However, gamma and other oscillation (vibration) frequencies that are effective in cognitive functions and produced by GABAergic neurons also decrease in autism. ${ }^{[51,52]}$ If we want to examine the biological effects of the damage of this system from a different perspective, we can evaluate the effect of the drug Fenobarbital in epilepsy. Phenobarbital, when used for severe seizures in epilepsy, paradoxically aggravates electroencephalogram discharges and is associated with high complication rates, suggesting that GABA can stimulate neurons rather than inhibiting epilepsy patients. In epileptic neurons, elevated levels of intracellular chloride [(Cl-) i] (as in autism), which shift the polarity of GABA's effects from inhibition to excitation, and the underlying paradoxical effects of phenobarbital may be this change. ${ }^{[53]}$ Summarized observations suggest that reducing intracellular chloride concentration may provide a useful treatment for autism. ${ }^{[26]}$ 
As can be seen, many different pathways can be targeted for autism treatment and treatment mechanisms can be developed for them. In this review, animal studies and clinical studies on how bumetanide affects autism and related behaviors through the GABAergic system are tried to be summarized.

\section{Intracellular Chloride Concentration and Autism Treatment}

The maturation of the brain; This occurs by the developmental sequential expression of voltagegated, receptor synapse-guided channels, and the developmental changes of the effects of the inhibitory transmitter GABA. For example, immature neurons have higher $[(\mathrm{Cl}-) \mathrm{i}]$ than adults. ${ }^{[54,55]}$ Because the gene encoding chloride import co-carrier NKCC1 is expressed early, while the chloride export KCC2 operates later. The expression of cation-chloride exporter KCC2 decreases before and immediately after birth. Subsequently, as the development continues, the intracellular chloride concentration is reduced by upregulation of $\mathrm{KCC} 2$. This regulation mechanism of $[(\mathrm{Cl}-)$ i] can also vary with various lesions, seizures and neurological disorders (eg OSB) so that the effect of GABA can change from inhibitor to excitator. ${ }^{[56,57]}$

Regulating $[(\mathrm{Cl}-) \mathrm{i}]$ level in the treatment of autism and returning it to normal is a treatment strategy and NKCC1 is generally targeted in the studies because KCC2 is labile and currently does not have a selective agonist. In contrast, NKCC1 is stable, its antagonists are identified, the diuretic Bumetanide is an antagonist that is highly specific to NKCC $1 .{ }^{[58]}$ For this reason, studies focused on boumethane in order to produce pharmacological treatment in autism.

\section{Animal Models}

In recent years, various animal models of ASD have been created to identify the molecular and cellular mechanisms underlying autism spectrum disorders and to develop new therapeutic tools. For this purpose, genetic disorders detected in ASD patients were included in the genome of mice and genetically based models were created. ${ }^{[57]}$ Also, an autism model was created with environmental factors such as exposure to valproic acid (VPA), which is known to be a risk factor for autism during pregnancy. ${ }^{[59]}$

In accordance with observations on humans, animal studies have also revealed dysfunctions in GABAergic signals. ${ }^{[57]}$ Studies show that GABA generates autism- like stereotypes in animal models when there is a dysfunction in the signal transmission. These animals are experimentally created by manipulating MECP2, TSC1 and TSC2 and FMR1 candidate genes. ${ }^{[4]}$ Fragile $X$ syndrome is a monogenetic disorder and the FMR1 gene causing the disease; It is characterized by mental disorders such as speech disorders, hyperactivity, autistic behavior and seizures. In mice without the FMR1 gene, an increase in network stimulation occurs in cortical circuits, including specific types of interneurons associated with excitation/inhibition (E/I) imbalance..$^{[57,60]}$ Another neurodevelopmental disorder with high co-morbidity with autism is Rett syndrome. This is due to mutations in the X-linked MECP2 gene. Cognitive disorders, speech loss, motor abnormalities, respiratory dysrhythmias, stereotypical behaviors and sometimes seizures that lead to premature death develop. Similar to FMR1 knock-out (KO) mice, these animals also show changes in the E/I balance. ${ }^{[57]} \mathrm{E} / \mathrm{I}$ imbalance; It has also been found in individuals with Tuberous sclerosis, a genetic multisystem disorder characterized by hamartomas spread in various organs including the brain, heart, skin, eyes, kidney, lung and liver. In patients with tuberous sclerosis, various neurological disorders such as retardation, autism-like disorders and epilepsy are observed in men.

The genes affected are here TSC1 and TSC2. ${ }^{[61]}$ Dravet syndrome and Angelman syndrome show similar characteristics to these models. Mutations in the En2 gene, which plays a role in the structure and neuronal differentiation of the midbrain/hindbrain region, have also been associated with ASD. En2 KO mice are also proposed as a model because they show similar behaviors to behavioral abnormalities observed in ASD patients. ${ }^{[57,62]}$

In addition to animal models created by genetic manipulation, there are also autism models created by applying medication. An example of this is the valproic acid model.

Valproic acid (VPA) is a commonly used medicine to treat epilepsy and bipolar disorders. Rat pups exposed to VPA in the uterus also show neurodevelopmental abnormalities and behavioral disorders similar to those with ASD. ${ }^{[63]}$ As with other animal models that exhibit OSB-like behavior, autistic phenotype; associated with changing $\mathrm{E} / \mathrm{l}$ balance. ${ }^{[64]}$

Animal studies are valuable in understanding common pathogenic pathways that lead to autism spectrum disorders, and animal models used for this purpose are also vital. Research on these models with 
the right organized studies has the potential to offer new biological treatment options. ${ }^{[4]}$

\section{Preclinical and Clinical Bumetanide Studies}

Bumetanide NKCC1 antagonist with a highly specific affinity is a reticent and reduces intracellular chlorine. In adults since 1975 and in children since 1986; It is used to treat hypertension, bronchopulmonary dysplasia, nephritic syndromes or heart congestion, and its pharmacokinetics is well known. ${ }^{[65]}$ The use of bumetanide in children is safe provided that it is accompanied by clinical and biological surveillance to control hypokalemia. ${ }^{[26]}$ However, Bumetanide converts GABA action from depolarization to hyperpolarization, and can also reduce cell excitability by regulating cell volume and extracellular space. ${ }^{[57,66]}$ With the effect of Bumetanide on GABA, its safety in humans (children) makes this drug the focus of its work for the treatment of ASD.

In recent years, on bumetanide autism animal models; It has been tried for reasons such as illuminating disease mechanisms and evaluating the effectiveness of possible treatments. In 2014, Tyzio et al. ${ }^{[67]}$ studied with VPA rats and Fragile $X$ mice. In these models, high chloride levels, increased GABA excitation, increased glutamatergic activity, and high gamma oscillations (oscillations) were detected during delivery at hippocampal neurons. When these models were treated with bumetanide shortly before birth, the stimulating effect of GABA was suppressed in both animal models and autisticlike behaviors were prevented. In 2015, Holmes et al. ${ }^{[68]}$ Assuming that early seizures will disrupt normal brain connectivity during brain maturation and cause autistic phenotype, normal rat pups after birth 5-14. repeated seizures induced by fluorotil between days 18 and 18-25. They subject subjects to a controlled test to observe the developmental changes of brain vibration activity between days. The results show that early life attacks change the development of brain vibrations and result in autistic-like behaviors. Bumetanide prevented abnormalities in coherence and voltage correlation with the application during seizures and improved the social seizure and gained a normal seizure threshold. When these preclinical studies are evaluated together, in rodent autism models; Chronic inadequate chloride regulation has been demonstrated, and the clinical effects of bumetanide have been confirmed.

Along with animal studies, numerous clinical studies have been conducted to evaluate the effect of bumetanide on autism. In 2010, Lemonnier et al. ${ }^{[56]}$
GABA has been suggested to play an important role in infantile autistic syndrome (IAS), and extensive research has shown that GABA's stimulating effects in neurological disorders are due to the steady increase of [(Cl-) i]. NKCC1 antagonist, which has decreased, tested the effects of bumetanide. During a 3-month period, five children (3-11 years) received $0.5 \mathrm{mg}$ of bumetanide twice daily. Then clinical and biological tests were carried out. As a result, an improvement in autistic behavior was observed without serious side effects. 5 standard IOS severity tests were used: Childhood Autism Rating Scale (CARS), Abnormal Behavior Checklist $(A B C)$, Clinical Global Impressions (CGI); Repetitive and Restrictive Behaviors (RRB) and Regulation Disorders Evaluation Grid (RDEG). In 2012, Lemonnier et al., ${ }^{[26]}$ this time, randomized grouping of 54 patients (3-11 years old) performed 3 months, $1 \mathrm{mg}$ of bumethane and placebo daily, followed by a one-month cleansing period. The CARS test showed a statistically significant treatment in scoring. Unlike placebo, those treated with boumetanide showed a moderate or mild $(<36.5)$ picture from severe condition (CARS >36.5), and there was a marked improvement in their way of communicating with their parents' observations. The therapeutic usefulness and benefit/risk ratio were also demonstrated by the commonly used CGI and there was a remarkable improvement with little side effects. As a side effect, only mild hypokalemia occurred in $30 \%$ of children and it was controlled by $\mathrm{K}+$ supplementation.

In a case report published in 2014, sensory behaviors, which are not one of the main symptoms of ASD but cause social dissonance, were tested for a long time (18 months). In this study, 10 year old girl with Asperger syndrome was evaluated after daily treatment of $2 \mathrm{mg}$ boometanite by dividing it into three using the Dunn Sense Profile. Biological tests were performed every month to assess the patient's health. Improvements observed after 18 months included a wide range of sensory behaviors (auditory, vestibular, tactile, multidimensional and oral sensory processing). Difficulties in sensory behaviors are an important determinant of social incompatibility, as they will cause incompatible emotional and physical reactions to environmental stimuli, though not yet among the main symptoms (eg, social interaction, communication disorders, stereotypical and restrictive behaviors) of ASD. ${ }^{[69]}$

In 2015, Hadjikhani et al. ${ }^{[58]}$ studied a 10-month open-label study of the efficacy and efficacy of bumetanitis in adults and young adults with ASD. They tested the effect of bumetanide therapy with 
functional magnetic resonance imaging (fMRI) on emotion recognition performance. This was done by evaluating changes in brain activation in response to the perception of dynamic films of facial expressions in two separate sessions before and after treatment. As a result, it has been revealed that bumetanide therapy improves emotion recognition and increases the activation of brain regions involved in social and emotional perception during emotional perception of faces. However, being open-labeled and containing 7 patients, two measurements divided by a long time interval, all participants having a normal intelligence are conditions that narrow the scope of the study. ${ }^{[58]}$

In 2015, Du et al. ${ }^{[70]}$ investigated the therapeutic effects of the combined treatment of bumetanide and applied behavior analysis (ABA) in children with autism. A total of 60 children, with an average of 4.5 years diagnosed with autism, were randomly divided into two groups: a single treatment group $(n=28)$ and a combined treatment group $(n=32)$. Oral bumetanide was given to the combined treatment group at $0.5 \mathrm{mg}$ twice daily with $A B A$ training. ABC, CARS and $C G I$ evaluations were performed just before and 3 months after the start of treatments. ABC and CGI total scores were significantly lower in the combined treatment group than in the single treatment group. $(p<0.05)$ No side effects of bumetanide were observed. As a result, bumetanide treatment together with $A B A$ education has been shown to result in a more beneficial treatment than children with $A B A$ education alone. A possible explanation for this is that bumetanide provides a cellular-level biological stability (eg, GABAergic inhibition), which makes the brain more sensitive to behavioral therapies. In order to reach a more comprehensive and secure judgment in this regard; A four-arm, double-blind, placebo-controlled long-term study consisting of a control group, a group receiving ABA (Applied Behavior Analysis), a group receiving boumetanite, and a group receiving $A B A$ and bumetanide together may be illuminating.

In 2017, Lemonnier et al. ${ }^{[71]}$ reported the results of the multicenter phase IIB study to evaluate the dose/ response and safety effects of bumetanide. A doubleblind, randomized, placebo-controlled, multi-site, dose-spaced study was performed to evaluate efficacy, safety, pharmacokinetics and optimal bumetanide dose in children and adolescents with ASD. The criteria used include CARS, Social Sensitivity Scale (SRS) and Clinical Global Impressions-Improvement (CGI-I) scale. 88 patients with ASD spread across the entire pediatric population (2-18 years) were sub-grouped in the four age groups and randomized to take either bumetanide $(0.5,1.0$ or $2.0 \mathrm{mg}$ twice daily) or placebo for 3 months. The most common side effects were hypokalemia, increased urine elimination, anorexia, dehydration and asthenia. Hypokalaemia occurred mainly at the beginning of treatment at doses of 1.0 and $2.0 \mathrm{mg}$ twice daily and gradually improved with oral potassium supplements. As a result, it has been demonstrated that bumetanide improves the main symptoms of ASD in all targeted age range and provides a beneficial benefit/risk ratio, especially at $1.0 \mathrm{mg}$ twice daily. The results also show that the frequency and severity of adverse events increase with dose, whereas there is no clear dose-response relationship for efficacy. A dose of $1 \mathrm{mg}$ twice daily was determined as the optimal dose between safety and efficacy.

In 2017, Hadjikhani et al. ${ }^{[72]}$ showed that eye contact in autism leads to an exaggerated increase in amygdala activation. However, in 2018, 9 people (21.4 \pm 5.4 years old) have been shown to have a normal level of amygdala activation with eye contact in autism as a result of intervention with 10-month bumetanide treatment. In accordance with previous clinical studies, these data also support the stimulating/inhibitory dysfunction hypothesis in autism, and show that bumetanide can improve certain aspects of social processes in autism. ${ }^{[73]}$

In 2019, Hajri et al. ${ }^{[74]} 29$ children with an average age of 7.9 years older than 5 years with autism disorder received $1 \mathrm{mg} /$ day of Bumatanide for 12 months, while clinical and biological evaluation was performed periodically during the treatment period, at the same time, autism spectrum disorder with CARS and CGI was performed at 3-month intervals. severity was evaluated. Sixteen children were excluded from the study for reasons such as hypokalemia appearance, intolerance to treatment, and inadequate efficacy, as reported by parents. As a result, there was a significant improvement between the 3rd and 6th months.

In 2020, Feng et al. ${ }^{[75]}$ planned to treat bumetanide and vitamin D3 to a baby who could not make eye contact for 30 months, as previous studies have shown that Vitamin D3 or bumetanide treatment can improve OSB basic symptoms. ${ }^{[76,77]}$ Since the patient's symptoms did not change after 6 months of Vitamin D supplementation, Vitamin D3 supplementation was discontinued at the request of the parent due to lack of effectiveness. Then, when the bumetanide was started, it was observed that the patient's symptoms 
improved after 1 month of bumetanide. This case report suggests that Vitamin D3 and bumetanide target different mechanisms in the pathogenesis of ASD. In addition, it is predicted that ASD can be divided into different subtypes, among which it is estimated that one subtype may respond to Vitamin D3 treatment and another subtype may respond to boumethane.

In 2020, Zhang et al. ${ }^{[78]}$ conducted a 3-month clinical trial on the bumetanide group (bumetanide therapy, $0.5 \mathrm{mg}$ twice daily) and 83 patients aged 3-6 randomized as a control group. The difference between these studies and the others is the attempt to search for clinical evidence on ASD. In this regard, they measured the inhibitory (GABA) and stimulating (glutamate, Glx) neurotransmitter concentrations of each patient in magnetic resonance spectroscopy (MRI) in the insular cortex (IC) and visual cortex (VC) that evaluated the results as the beginning and 3 months later. Compared with the control group, the bumetanide group showed a significant reduction in symptom severity, as observed in the total CARS score. However, improvement in clinical symptoms was confirmed by CGI. The GABA/Glx ratio in both IC and VC decreased faster in the 3-month period in the bumetanide group than in the control group. This decrease in IC is associated with symptom improvement in the bumetanide group. The study confirmed the clinical effectiveness of bumetanide in relieving the main symptoms of ASD patients in young children, and this is the first indication that this development is associated with a decrease in GABA/Glx ratios. Ultimately, this study suggests that the GABA/Glx ratio measured by MRS may provide a neuroimaging biomarker to evaluate the treatment efficacy for bumetanide. However, patients with similar symptom scores; The study should be supported with further research, as it shows different neuroimaging findings.

To summarize, in all clinical studies from 2010 to 2020 , researchers have conducted studies to support the positive effects of bumetanide on patients with ASD. They achieved similar results in different doses, in different age groups, at different time intervals in this regard. Some studies have been evaluated within the scope of different abilities such as sensory behaviors, emotion identification rather than the main symptoms of autism and an attempt has been made to link different biomarkers with the severity of autism symptoms. The most common common side effect of the drug was hypokalemia, and it was brought under control with supportive treatments.
Studies in the final analysis show that bumetanide is a promising complementary therapy for autism treatment.

\section{DISCUSSION AND CONCLUSION}

In general, studies with bumetanide recorded similar healing results, no serious side effects, and proven safety suggest that pharmacological treatment may soon be a part of standard treatment in autism. Although the treatments applied do not cure autism completely, the positive effects are sufficient to continue working with larger groups in order to test the effects of the drug on ASD. When we think about side effects, it is possible to see that the studies are in harmony with each other. The results showed that bumetanide is safe but linked to adverse events associated with diuresis and dehydration. Of course, the most common hypokalemia; Potassium supplementation can be managed clinically by dose titration and careful hydration of the patient. ${ }^{[71]}$ Since ASD is a developmental disorder, early pharmacological intervention is essential. This situation of children; side effects mean you will take medications that need to be carefully considered. Many drugs that affect GABAergic synapses can cause addiction or paradoxical reactions, as their brain circuits are still immature and GABA may have a depolarizing and stimulating effect that can extend to the late stages of development. ${ }^{[57]}$ Therefore, considering all risks, pharmacological tools should be designed with properly designed studies, use of appropriate animal models and ultimately with minimal risk.

One of the conditions to be considered in patients with ASD is clinical variability, patients experience different degrees of difficulty with different mental abilities. Examples of these areas are intellectual ability, attention, depression and other psychological disorders. Said clinical heterogeneity has made it difficult to develop pharmacological treatments for ASD. This variability also makes it difficult for researchers seeking biomarkers or genetic variation in common with most ASD patients. ${ }^{[27,78]}$

The number of patients with ASD is limited, there are no consistent repetitions, the majority of studies are relatively short-term, and therefore there is no strong evidence of long-term effects, evaluation criteria; Lack of important situations such as intelligence assessment, school adaptation and comorbidity constitute the limitations of the mentioned studies. Although bumetanide studies evaluate the effectiveness of boumetanide in autism 
from many different aspects, there is still a need for more comprehensive double-blind, multicenter randomized and controlled studies.

\section{Declaration of conflicting interests}

The authors declared no conflicts of interest with respect to the authorship and/or publication of this article.

\section{Funding}

The authors received no financial support for the research and/or authorship of this article.

\section{REFERENCES}

1. Wingate M. Prevalence of autism spectrum disordersautism and developmental disabilities monitoring network, 14 sites, United States, 2008. MMWR Surveill Summ 2012;61:1-19.

2. Persico AM, Bourgeron T. Searching for ways out of the autism maze: genetic, epigenetic and environmental clues. Trends Neurosci 2006;29:349-58.

3. Krey JF, Dolmetsch RE. Molecular mechanisms of autism: a possible role for $\mathrm{Ca} 2+$ signaling. Curr Opin Neurobiol 2007;17:112-9.

4. Benvenuto A, Battan B, Porfirio MC, Curatolo P. Pharmacotherapy of autism spectrum disorders. Brain Dev 2013;35:119-27.

5. Breitenkamp AF, Matthes J, Herzig S. Voltage-gated Calcium Channels and Autism Spectrum Disorders. Curr Mol Pharmacol 2015;8:123-32.

6. Lord C, Elsabbagh M, Baird G, Veenstra-Vanderweele J. Autism spectrum disorder. Lancet 2018;392:508-20.

7. Hviid A, Stellfeld M, Wohlfahrt J, Melbye M. Association between thimerosal-containing vaccine and autism. JAMA 2003;290:1763-6.

8. Parker SK, Schwartz B, Todd J, Pickering LK. Thimerosalcontaining vaccines and autistic spectrum disorder: a critical review of published original data. Pediatrics 2004;114:793-804.

9. Christianson AL, Chesler N, Kromberg JG. Fetal valproate syndrome: clinical and neuro-developmental features in two sibling pairs. Dev Med Child Neurol 1994;36:361-9.

10. Schmidt RJ, Tancredi DJ, Ozonoff S, Hansen RL, Hartiala $\mathrm{J}$, Allayee $\mathrm{H}$, et al. Maternal periconceptional folic acid intake and risk of autism spectrum disorders and developmental delay in the CHARGE (CHildhood Autism Risks from Genetics and Environment) case-control study. Am J Clin Nutr 2012;96:80-9.

11. Rane P, Cochran D, Hodge SM, Haselgrove C, Kennedy DN, Frazier JA. Connectivity in Autism: A Review of MRI Connectivity Studies. Harv Rev Psychiatry 2015;23:223-44.

12. O'Reilly $C$, Lewis JD, Elsabbagh M. Is functional brain connectivity atypical in autism? A systematic review of EEG and MEG studies. PLoS One 2017;12:e0175870.

13. Menold MM, Shao Y, Wolpert CM, Donnelly SL, Raiford $\mathrm{KL}$, Martin ER, et al. Association analysis of chromosome 15 gabaa receptor subunit genes in autistic disorder. J Neurogenet 2001;15:245-59.
14. Dykens EM, Sutcliffe JS, Levitt P. Autism and 15q11-q13 disorders: behavioral, genetic, and pathophysiological issues. Ment Retard Dev Disabil Res Rev 2004;10:284-91.

15. Friocourt G, Poirier K, Rakić S, Parnavelas JG, Chelly J. The role of ARX in cortical development. Eur J Neurosci 2006;23:869-76.

16. Turner $G$, Partington $M$, Kerr B, Mangelsdorf $M$, Gecz J. Variable expression of mental retardation, autism, seizures, and dystonic hand movements in two families with an identical ARX gene mutation. Am J Med Genet 2002;112:405-11.

17. Campbell DB, Sutcliffe JS, Ebert PJ, Militerni R, Bravaccio $C$, Trillo $S$, et al. A genetic variant that disrupts MET transcription is associated with autism. Proc Natl Acad Sci U S A 2006;103:16834-9.

18. Birchmeier C, Gherardi E. Developmental roles of HGF/ SF and its receptor, the c-Met tyrosine kinase. Trends Cell Biol 1998;8:404-10.

19. Rossignol E. Genetics and function of neocortical GABAergic interneurons in neurodevelopmental disorders. Neural Plast 2011;2011:649325.

20. Association AP. Diagnostic and statistical manual of mental disorders (DSM- $\left.{ }^{\oplus}\right)$. Washington: American Psychiatric Pub.; 2013.

21. Moon SJ, Hwang JS, Shin AL, Kim JY, Bae SM, SheehyKnight J, et al. Accuracy of the Childhood Autism Rating Scale: a systematic review and meta-analysis. Dev Med Child Neurol 2019;61:1030-8.

22. Bouvard M. Liste des comportements aberrants-version traduite. Issy Les Moulineaux: EAP/ECPA; 2000.

23. Busner J, Targum SD. The clinical global impressions scale: applying a research tool in clinical practice. Psychiatry (Edgmont) 2007;4:28-37.

24. Bourreau Y, Roux S, Gomot M, Barthélémy C. Repetitive and restricted behaviours (RRB) in autism: clinical evaluation. Encephale 2009;35:340-6.

25. Adrien JL, Rossignol-Deletang N, Martineau J, Couturier G, Barthelemy C. Regulation of cognitive activity and early communication development in young autistic, mentally retarded, and young normal children. Dev Psychobiol 2001;39:124-36.

26. Lemonnier E, Degrez C, Phelep M, Tyzio R, Josse F, Grandgeorge M, et al. A randomised controlled trial of bumetanide in the treatment of autism in children. Transl Psychiatry 2012;2:e202.

27. Chadman KK, Fernandes S, DiLiberto E, Feingold R. Do animal models hold value in Autism spectrum disorder (ASD) drug discovery? Expert Opin Drug Discov 2019;14:727-34.

28. Silverman JL, Pride MC, Hayes JE, Puhger KR, ButlerStruben HM, Baker S, et al. GABAB Receptor Agonist R-Baclofen Reverses Social Deficits and Reduces Repetitive Behavior in Two Mouse Models of Autism. Neuropsychopharmacology 2015;40:2228-39.

29. Veenstra-VanderWeele J, Cook EH, King BH, Zarevics $\mathrm{P}$, Cherubini M, Walton-Bowen $\mathrm{K}$, et al. Arbaclofen in Children and Adolescents with Autism Spectrum Disorder: A Randomized, Controlled, Phase 2 Trial. Neuropsychopharmacology 2017;42:1390-8. 
30. Ferreira-Chamorro P, Redondo A, Riego G, Leánez S, Pol O. Sulforaphane Inhibited the Nociceptive Responses, Anxiety- and Depressive-Like Behaviors Associated With Neuropathic Pain and Improved the Anti-allodynic Effects of Morphine in Mice. Front Pharmacol 2018;9:1332.

31. Singh K, Connors SL, Macklin EA, Smith KD, Fahey JW, Talalay $\mathrm{P}$, et al. Sulforaphane treatment of autism spectrum disorder (ASD). Proc Natl Acad Sci U S A 2014;111:15550-5.

32. Anagnostou E, Aman MG, Handen BL, Sanders KB, Shui A, Hollway JA, et al. Metformin for Treatment of Overweight Induced by Atypical Antipsychotic Medication in Young People With Autism Spectrum Disorder: A Randomized Clinical Trial. JAMA Psychiatry 2016;73:928-37.

33. Hazell P. Drug therapy for attention-deficit/hyperactivity disorder-like symptoms in autistic disorder. J Paediatr Child Health 2007;43:19-24.

34. Scahill L, Aman MG, McDougle CJ, McCracken JT, Tierney E, Dziura J, et al. A prospective open trial of guanfacine in children with pervasive developmental disorders. J Child Adolesc Psychopharmacol 2006;16:589-98.

35. Miano S, Ferri R. Epidemiology and management of insomnia in children with autistic spectrum disorders. Paediatr Drugs 2010;12:75-84.

36. Malow B, Adkins KW, McGrew SG, Wang L, Goldman $\mathrm{SE}$, Fawkes D, et al. Melatonin for sleep in children with autism: a controlled trial examining dose, tolerability, and outcomes. J Autism Dev Disord 2012;42:1729-37.

37. Ming X, Gordon E, Kang N, Wagner GC. Use of clonidine in children with autism spectrum disorders. Brain Dev 2008;30:454-60.

38. Zafeiriou DI, Ververi A, Vargiami E. The serotonergic system: its role in pathogenesis and early developmental treatment of autism. Curr Neuropharmacol 2009;7:150-7.

39. Ernst M, Zametkin AJ, Matochik JA, Pascualvaca D, Cohen RM. Low medial prefrontal dopaminergic activity in autistic children. Lancet 1997;350:638.

40. Mori T, Mori K, Fujii E, Toda Y, Miyazaki M, Harada M, et al. Evaluation of the GABAergic nervous system in autistic brain: (123)l-iomazenil SPECT study. Brain Dev 2012;34:648-54.

41. Choudhury PR, Lahiri S, Rajamma U. Glutamate mediated signaling in the pathophysiology of autism spectrum disorders. Pharmacol Biochem Behav 2012;100:841-9.

42. Maneeton N, Maneeton B, Putthisri S, Woottiluk $P$, Narkpongphun A, Srisurapanont M. Risperidone for children and adolescents with autism spectrum disorder: a systematic review. Neuropsychiatr Dis Treat 2018;14:1811-20.

43. King $B H$, Wright DM, Handen BL, Sikich L, Zimmerman AW, McMahon W, et al. Double-blind, placebo-controlled study of amantadine hydrochloride in the treatment of children with autistic disorder. J Am Acad Child Adolesc Psychiatry 2001;40:658-65.

44. Green JJ, Hollander E. Autism and oxytocin: new developments in translational approaches to therapeutics. Neurotherapeutics 2010;7:250-7.
45. Guastella AJ, Einfeld SL, Gray KM, Rinehart NJ, Tonge BJ, Lambert TJ, et al. Intranasal oxytocin improves emotion recognition for youth with autism spectrum disorders. Biol Psychiatry 2010;67:692-4.

46. Palmieri L, Papaleo V, Porcelli V, Scarcia P, Gaita L, Sacco $\mathrm{R}$, et al. Altered calcium homeostasis in autism-spectrum disorders: evidence from biochemical and genetic studies of the mitochondrial aspartate/glutamate carrier AGC1. Mol Psychiatry 2010;15:38-52.

47. Brown MJ, Willis T, Omalu B, Leiker R. Deaths resulting from hypocalcemia after administration of edetate disodium: 2003-2005. Pediatrics 2006;118:e534-6.

48. Sinha Y, Silove N, Williams K. Chelation therapy and autism. BMJ 2006;333:756.

49. Ghanizadeh A. Can ziconotide as a N-type voltagesensitive calcium channel blocker open a new mode for treatment of autism? A hypothesis. Neurosciences (Riyadh) 2011;16:83.

50. Pizzarelli R, Cherubini E. Alterations of GABAergic signaling in autism spectrum disorders. Neural Plast 2011;2011:297153.

51. Wilson TW, Rojas DC, Reite ML, Teale PD, Rogers SJ. Children and adolescents with autism exhibit reduced MEG steady-state gamma responses. Biol Psychiatry 2007;62:192-7.

52. Brown C, Gruber T, Boucher J, Rippon G, Brock J. Gamma abnormalities during perception of illusory figures in autism. Cortex 2005;41:364-76.

53. Nardou R, Yamamoto S, Chazal G, Bhar A, Ferrand $\mathrm{N}$, Dulac $\mathrm{O}$, et al. Neuronal chloride accumulation and excitatory GABA underlie aggravation of neonatal epileptiform activities by phenobarbital. Brain 2011;134:987-1002.

54. Spitzer NC, Gu X, Olson E. Action potentials, calcium transients and the control of differentiation of excitable cells. Curr Opin Neurobiol 1994;4:70-7.

55. Ben-Ari Y, Gaiarsa JL, Tyzio R, Khazipov R. GABA: a pioneer transmitter that excites immature neurons and generates primitive oscillations. Physiol Rev 2007;87:1215-84.

56. Lemonnier E, Ben-Ari Y. The diuretic bumetanide decreases autistic behaviour in five infants treated during 3 months with no side effects. Acta Paediatr 2010;99:1885-8.

57. Cellot G, Cherubini E. GABAergic signaling as therapeutic target for autism spectrum disorders. Front Pediatr 2014;2:70.

58. Hadjikhani N, Zürcher NR, Rogier O, Ruest T, Hippolyte $\mathrm{L}$, Ben-Ari $\mathrm{Y}$, et al. Improving emotional face perception in autism with diuretic bumetanide: a proof-of-concept behavioral and functional brain imaging pilot study. Autism 2015;19:149-57.

59. Meador KJ, Loring DW. Risks of in utero exposure to valproate. JAMA 2013;309:1730-1.

60. Gonçalves JT, Anstey JE, Golshani P, Portera-Cailliau C. Circuit level defects in the developing neocortex of Fragile X mice. Nat Neurosci 2013;16:903-9.

61. Curatolo P, Bombardieri R. Tuberous sclerosis. Handbook of Clinical Neurology 2008;87:129-51. 
62. Cheh MA, Millonig JH, Roselli LM, Ming X, Jacobsen E, Kamdar S, et al. En2 knockout mice display neurobehavioral and neurochemical alterations relevant to autism spectrum disorder. Brain Res 2006;1116:166-76.

63. Nicolini C, Fahnestock M. The valproic acid-induced rodent model of autism. Exp Neurol 2018;299:217-27.

64. Banerjee A, García-Oscos F, Roychowdhury S, Galindo LC, Hall S, Kilgard MP, et al. Impairment of cortical GABAergic synaptic transmission in an environmental rat model of autism. Int J Neuropsychopharmacol 2013;16:1309-18.

65. Sullivan JE, Witte MK, Yamashita TS, Myers CM, Blumer JL. Dose-ranging evaluation of bumetanide pharmacodynamics in critically ill infants. Clin Pharmacol Ther 1996;60:424-34.

66. Hochman DW. The extracellular space and epileptic activity in the adult brain: explaining the antiepileptic effects of furosemide and bumetanide. Epilepsia 2012;53 Suppl 1:18-25.

67. Tyzio R, Nardou R, Ferrari DC, Tsintsadze T, Shahrokhi A, Eftekhari $S$, et al. Oxytocin-mediated GABA inhibition during delivery attenuates autism pathogenesis in rodent offspring. Science 2014;343:675-9.

68. Holmes GL, Tian C, Hernan AE, Flynn S, Camp D, Barry J. Alterations in sociability and functional brain connectivity caused by early-life seizures are prevented by bumetanide. Neurobiol Dis 2015;77:204-19.

69. Grandgeorge $M$, Lemonnier E, Degrez C, Jallot N. The effect of bumetanide treatment on the sensory behaviours of a young girl with Asperger syndrome. BMJ Case Rep 2014;2014:bcr2013202092.

70. Du L, Shan L, Wang B, Li H, Xu Z, Staal WG, et al. A Pilot Study on the Combination of Applied Behavior Analysis and Bumetanide Treatment for Children with Autism. J
Child Adolesc Psychopharmacol 2015;25:585-8.

71. Lemonnier E, Villeneuve N, Sonie S, Serret S, Rosier A, Roue $M$, et al. Effects of bumetanide on neurobehavioral function in children and adolescents with autism spectrum disorders. Transl Psychiatry 2017;7:e1056.

72. Hadjikhani N, Åsberg Johnels J, Zürcher NR, Lassalle A, Guillon Q, Hippolyte L, et al. Look me in the eyes: constraining gaze in the eye-region provokes abnormally high subcortical activation in autism. Sci Rep 2017;7:3163.

73. Hadjikhani N, Åsberg Johnels J, Lassalle A, Zürcher NR, Hippolyte L, Gillberg C, et al. Bumetanide for autism: more eye contact, less amygdala activation. Sci Rep 2018;8:3602.

74. Hajri M, Ben Amor A, Abbes Z, Dhouib S, Ouanes S, Mrabet $A$, et al. Bumetanide in the management of autism. Tunisian experience in Razi Hospital. Tunis Med 2019;97:971-7.

75. Feng JY, Li HH, Wang B, Shan L, Jia FY. Successive clinical application of vitamin $D$ and bumetanide in children with autism spectrum disorder: A case report. Medicine (Baltimore) 2020;99:e18661.

76. Jia F, Wang B, Shan L, Xu Z, Staal WG, Du L. Core symptoms of autism improved after vitamin $D$ supplementation. Pediatrics 2015;135:e196-8.

77. Feng J, Shan L, Du L, Wang B, Li H, Wang W, et al. Clinical improvement following vitamin D3 supplementation in Autism Spectrum Disorder. Nutr Neurosci 2017;20:284-90.

78. Zhang L, Huang CC, Dai Y, Luo Q, Ji Y, Wang K, et al. Symptom improvement in children with autism spectrum disorder following bumetanide administration is associated with decreased GABA/glutamate ratios. Transl Psychiatry 2020;10:9. 